EPJ Web of Conferences 40, 17003 (2013)

DOI: $10.1051 /$ epjconf/20134017003

(c) Owned by the authors, published by EDP Sciences, 2013

\title{
Magnetoabsorption and magnetic hysteresis in Ni ferrite nanoparticles
}

\author{
P. Hernández-Gómez ${ }^{1}$, J. M. Muñoz ${ }^{1}$, M. A. Valente ${ }^{2}$, C. Torres ${ }^{1}$, and C. de Francisco ${ }^{1}$ \\ ${ }^{1}$ Dpt. Electricidad y Electrónica, Univ. Valladolid, 47011 Valladolid, Spain \\ ${ }^{2}$ Dpt. Fisica, Univ. Aveiro, Aveiro, Portugal
}

\begin{abstract}
Nickel ferrite nanoparticles were prepared by a modified sol-gel technique employing coconut oil, and then annealed at different temperatures in $400-1200{ }^{\circ} \mathrm{C}$ range. This route of preparation has revealed to be one efficient and cheap technique to obtain high quality nickel ferrite nanosized powder. Sample particles sizes obtained with XRD data and Scherrer's formula lie in $13 \mathrm{~nm}$ to $138 \mathrm{~nm}$, with increased size with annealing temperature. Hysteresis loops have been obtained at room temperature with an inductive method. Magnetic field induced microwave absorption in nanoscale ferrites is a recent an active area of research, in order to characterize and explore potential novel applications. In the present work microwave magnetoabsorption data of the annealed nickel ferrite nanoparticles are presented. These data have been obtained with a system based on a network analyzer that operates in the frequency range $0-8.5 \mathrm{GHz}$. At fields up to $400 \mathrm{mT}$ we can observe a peak according to ferromagnetic resonance theory. Sample annealed at higher temperature exhibits different absorption, coercivity and saturation magnetization figures, revealing its multidomain character.
\end{abstract}

\section{Introduction}

Spinel ferrites are widely used for electrotechnical equipment since their discovery in the forties, due their combination of useful magnetic properties, the possibility of tailor them with appropriate cation substitutions, the reduced electrical conductivity regarding ferromagnetic alloys, and their easy and inexpensive ways to manufacture them. Nanoscale devices with engineered physical and magnetic properties result in substantial improvement in the quality of materials that are useful in a variety of applications. They are interesting due to their size-dependent optical, electronic, magnetic, thermal, mechanical, and chemical properties. In the last years there is a strong interest in ferrite nanoparticles due to the fact that nanoscale materials possess properties that are comparable to, or superior to those of bulk materials counterparts [1], to the possibility of analyzing the fundamental aspects of magnetic ordering phenomena in magnetic materials with reduced dimensions [2], as well as its potential applications in high density data storage, magnetic sensors, spintronics, magnetic resonance imaging, ferrofluids, magnetically guided drug delivery, or microwave devices[3].

High frequency microwave absorption of magnetic materials is an interesting topic in order to obtain new materials for use as radar absorbing materials, but they are also interesting in the field of wireless communications. Among spinel ferrites, nickel based ferrites have been employed as electromagnetic wave absorbing material in UHF, VHF and X bands [4-6]. Magnetic field induced microwave absorption in nanoscale ferrites is a recent and active area of research, in order to characterize and explore potential novel applications, like acoustic modulators or sensitive field sensors [7].

\section{Experimental setup}

\subsection{Sample preparation}

Nickel ferrite nanoparticles were prepared by a modified sol-gel technique, with starting components $\mathrm{Fe}\left(\mathrm{NO}_{3}\right)_{3} \cdot 9 \mathrm{H}_{2} \mathrm{O}$ (iron (III) nitrate nonahydrate), $\mathrm{Ni}\left(\mathrm{NO}_{3}\right)_{2} \cdot 6 \mathrm{H}_{2} \mathrm{O}$ (nickel (II) nitrate hexahydrate) and lyophilized coconut oil. This route of preparation has revealed to be one efficient and cheap technique to obtain high quality nickel ferrite nanosized powder [8] The nitrates were dissolved in a suspension of coconut oil, prepared with a molar concentration highly superior to the corresponding to critical micelle concentration, that is $8.1 \mathrm{mmol} / \mathrm{l}$. Then, water excess is removed by keeping the suspension at $75{ }^{\circ} \mathrm{C}$ during $24 \mathrm{~h}$. After that, the dried samples were annealed in air at different temperatures: 400, 600, 800, 1000 and $1200{ }^{\circ} \mathrm{C}$ during $4 \mathrm{~h}$. Crystallization is obtained by fast cooling to room temperature. Finally, the samples were milled in agate mortar. XRD analysis gives particle sizes: 13, 42, 75, 
120, and $138 \mathrm{~nm}$ resp., with single phase nickel ferrite formation [9].

\subsection{Experimental techniques}

\subsubsection{Magnetic Hysteresis measurements}

Magnetic hysteresis loops have been obtained with a robust and digitally processed system developed in our laboratory, based in the induced voltages in two identical coils, one of them filled with the nanoparticle powders. The $50 \mathrm{~Hz}$ alternating magnetic field with amplitude up to $4 \mathrm{kOe}$ is produced with an self transformer which feeds the primary coil of 1200 turns. Magnetic flux is driven to secondary coils with the help of a ferromagnetic closed magnetic circuit. Current in the primary circuit, and the induced voltages in secondary coils are sent to computer with the help of a USB data logger Agilent U2542A that captures up to $500 \mathrm{KSa} / \mathrm{s}$ with 16 bit resolution. M-H hysteresis loops are then calculated in the control program generated with Agilent VEE software, which operates as a virtual instrument. With this measurement setup we are able to perform a fast and accurate measurement of room temperature hysteresis loops of soft magnetic materials.

\subsubsection{Microwave magnetoabsorption measurements}

Magnetic field induced microwave absorption has been obtained with the help of an automatic measuring system based on a network analyzer Agilent model E5071C working up to $8.5 \mathrm{GHz}$. The sampleholder is placed into the polar pieces of an electromagnet which produce magnetic fields up to $6 \mathrm{kOe}$, measured with a gaussmeter FWBell 5080 with a calibrated Hall probe. All the system is controlled with a PC with an appropriate Agilent VEE control program. The powdered sample is located in an area with minimum $\mathrm{rf}$ electric field and maximum $\mathrm{rf}$ magnetic field. In addition, this setup allow the broadband measurement of microwave absorption with varying continuously both the operating frequency and DC magnetic field. Details of this system can be found in ref 9 .

\section{Results and discussion}

M-H magnetic hysteresis loops of the nickel ferrite nanoparticle samples analyzed are represented in the figure 1. We can observe different behaviour of the samples: for the intermediate annealing temperatures we obtain very similar loops, with a maximum coercive field of $171 \mathrm{Oe}$ for the sample annealed at $800{ }^{\circ} \mathrm{C}$. On the contrary, the sample annealed at $1200{ }^{\circ} \mathrm{C}$ has a top saturation magnetization of about $40 \mathrm{emu} / \mathrm{g}$, higher than other samples, but the coercive field is strongly reduced to 52 Oe. Details of magnetic parameters obtained from hysteresis loops are depicted in the figure 2 . and also in the Table 1 The increase in saturation magnetization with annealing temperature is consistent with the improvement in crystallinity [2], whereas remanence is rather similar in all the analyzed samples, around $8 \mathrm{emu} / \mathrm{g}$. Values of saturation magnetization are rather lower to the value corresponding to bulk ferrite $(56 \mathrm{emu} / \mathrm{g}$ ) pointing to some degree of non collinear spin structure usually explained with a core-shell particle model [10]. Regarding coercive field, it is clear that over $800{ }^{\circ} \mathrm{C}$ it diminishes strongly Figures expressed in Table 1 are in the same order of magnitude that analog spinel ferrite nanoparticles or $\mathrm{Ni}$ ferrite synthetized with other methods [11-14]

Finally, the sample with the lower annealing temperature studied $\left(400{ }^{\circ} \mathrm{C}\right)$ exhibits a qualitatively different hysteresis loop, with non attainment of saturation, that can be understood as the onset of superparamagnetic behaviour [10], in which the best model is a core shell morphology with a ferrimagnetic core and a surface layer on the shell with broken superexchange bonds between magnetic cations that induce a large surface spin disorder, so that , any increase in the applied field has an effect only on the surface layer of the particles. Since this layer does not have a very high response to the applied field, the overall increase in the magnetization of the particles slows down, and hence a high field magnetization irreversibility occurs.

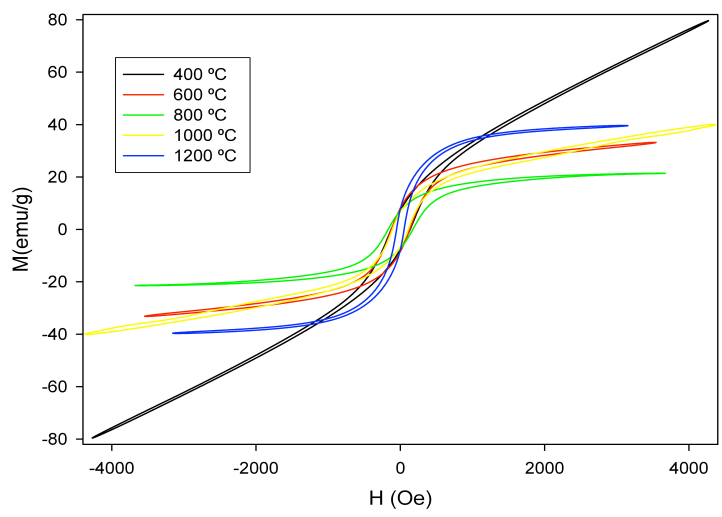

Fig. 1. Hysteresis loops of Ni ferrite nanoparticles.

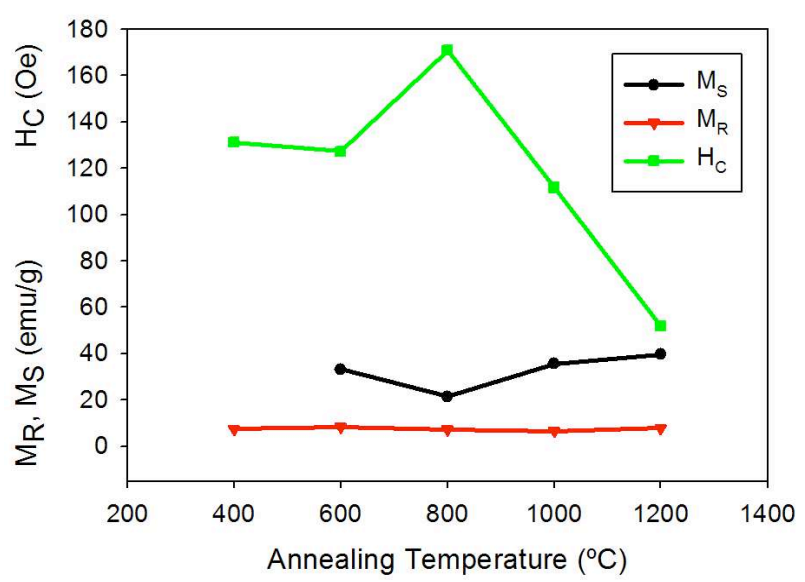

Fig. 2. Coercive field, saturation magnetization and remanence of Ni ferrite nanoparticles. 
Table 1. Magnetic parameters of Ni ferrite nanoparticles.

\begin{tabular}{|c|c|c|c|}
\hline $\begin{array}{c}\text { Annealing } \\
\text { temp }\end{array}$ & $\mathbf{M}_{\mathbf{S}}(\mathbf{e m u} / \mathbf{g})$ & $\mathbf{M}_{\mathbf{R}}(\mathbf{e m u} / \mathbf{g})$ & $\mathbf{H}_{\mathbf{C}}(\mathbf{O e})$ \\
\hline $400^{\circ} \mathrm{C}$ & - & 7.5 & 131.2 \\
$600^{\circ} \mathrm{C}$ & 33.2 & 8.3 & 127.3 \\
$800^{\circ} \mathrm{C}$ & 21.4 & 7.2 & 170.8 \\
$1000^{\circ} \mathrm{C}$ & 35.7 & 6.6 & 111.6 \\
$1200^{\circ} \mathrm{C}$ & 39.6 & 7.8 & 51.9 \\
\hline
\end{tabular}

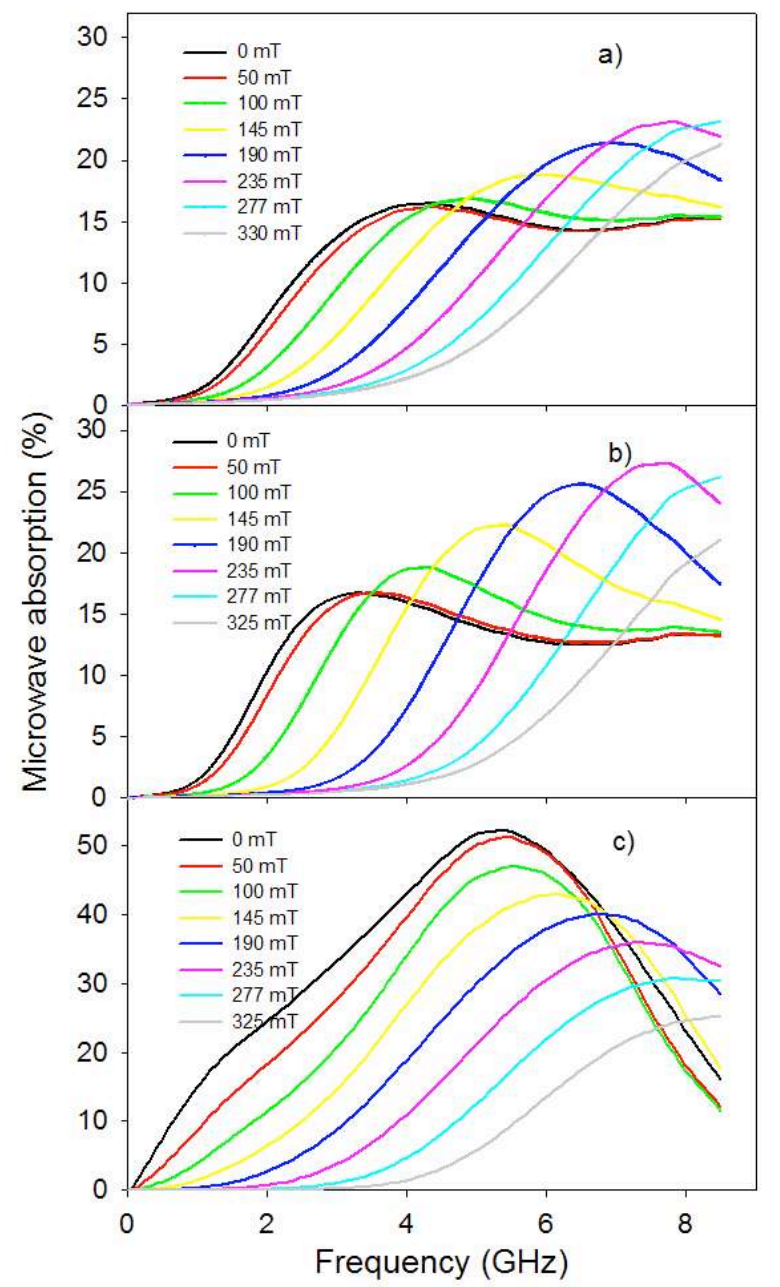

Fig. 3. Microwave absorption as a function of frequency, at different applied magnetic fields (curves shift with increasing applied field from left to right in each graph), corresponding to $\mathrm{NiFe}_{2} \mathrm{O}_{4}$ nanoparticles annealed at a) $400{ }^{\circ} \mathrm{C}$, b) $800^{\circ} \mathrm{C}$, and c) $1200{ }^{\circ} \mathrm{C}$.

A representative set of magnetic field induced microwave absorption spectra of the analyzed ferrite system are represented in the figure 3 . We can see that for each annealing temperature we observe a single peak of maximum absorption that shifts to higher frequencies with the increase of the applied DC magnetic field. These peaks are very broad in frequency, probably due to size dispersion, so that they could be good candidates to microwave absorbers. Taking into account the effect of annealing temperature, we can state that the maximum absorption increases slightly with the annealing temperature, with an absorption of about $25 \%$, for all the samples annealed below $1000{ }^{\circ} \mathrm{C}$. On the other hand, the end member of the series, annealed at $1200{ }^{\circ} \mathrm{C}$, exhibits different behaviour, with a qualitatively different absorption profile, with maximum absorption at zero field regarding the other samples, an increased absorption to figures over $50 \%$, and with a broader absorption curve, i.e. the best properties in the samples studied.

In these data it is also noteworthy the existence of zero field absorption. As explained in 2.2.2, sample is placed in the area with maximum rf magnetic field, however, low field absorption due to conduction mechanisms in particles are probably present in our samples. This absorption is caused by induced electric fields originated by alternating magnetic signal $[15,16]$ and has non resonant characteristics, so that at low DC field we are observing the effect of both non resonant low frequency absorption and resonance caused by internal fields as we describe below.

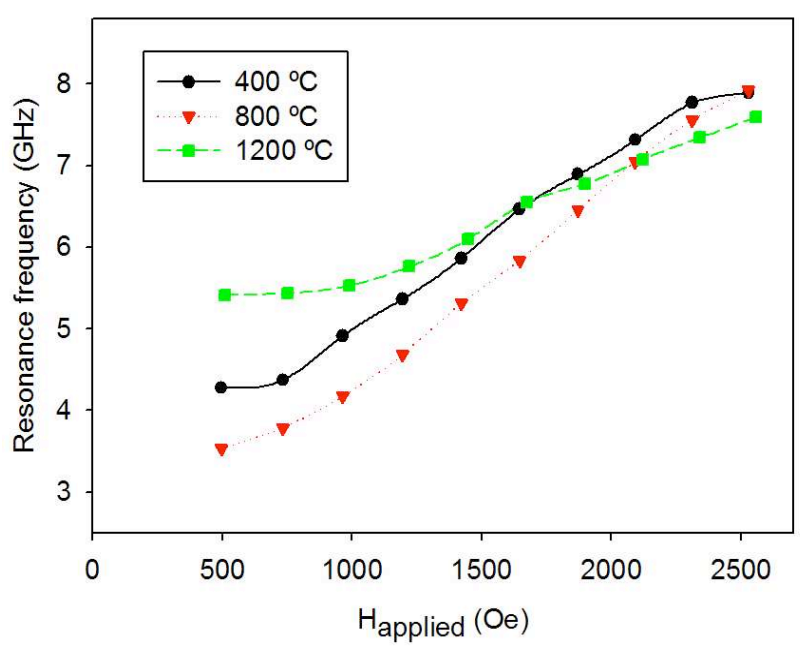

Fig. 4 Resonance frequency as a function of applied field, corresponding to $\mathrm{NiFe}_{2} \mathrm{O}_{4}$ nanoparticles

These results have been deeply analyzed in a preceding paper [9], and the main finding was that: the results agree well with the theory of ferromagnetic resonance. Plots of resonance frequency versus DC applied field (represented in figure 4) give in general linear results corresponding to g-factors in the range 2.12 to 2.21 , in good agreement with literature data for $\mathrm{Ni}$ ferrites [7]. A closer inspection reveals that we are dealing again with three different behaviour: at intermediate annealing temperatures we obtain very similar results, up to the sample annealed at $1000{ }^{\circ} \mathrm{C}$ that marks the onset of a different behaviour, which sample annealed at $1200{ }^{\circ} \mathrm{C}$ exhibit clearly. In this case the slope 
of the line is different probably due to the fact that its particle size is big enough to allow multidomain structure, and hence the resonance phenomena is composed by spin as well as domain wall resonances, the latter occurring at lower frequencies. The composition of these two resonance mechanisms will broaden and distort the resonance line as observed. On the other hand, $400^{\circ}$ $\mathrm{C}$ sample has the same characteristics of the intermediate annealed temperature samples, but exhibit a higher resonance frequency, thus indicating a higher internal field and hence an increased anisotropy [15], in accordance with hysteresis results. As explained above, non resonant low field absorption distort the resonance line at lower DC applied fields, so that figures obtained below $1 \mathrm{kOe}$ [16] have not been accounted for $\mathrm{g}$ - factor calculations.

Keeping in mind all the data presented above, we can state that all the system analyzed is in ferrimagnetic region at room temperature, unless the lower annealing temperature studied promote a particle size near to the superparamagnetic limit, as observed in hysteresis loops, unless this fact has no remarkable effect on microwave magnetoabsorption regarding the other samples analyzed. At intermediate annealing temperatures the behaviour is similar, with small variations in both magnetic parameters and absorption figures, being the sample annealed at $800{ }^{\circ} \mathrm{C}$ the one with best coercive field. Sample annealed at $1000{ }^{\circ} \mathrm{C}$ marks the onset of multidomain formation, clearly indicated by the sudden diminution of coercive field, which is remarkable in the sample annealed at $1200{ }^{\circ} \mathrm{C}$, and also observed in different microwave magnetoabsorption spectrum. Preliminary measurements in magnetic relaxation (not presented in this work) also point to the multidomain character of this sample, whose relaxation spectrum is very similar to the bulk counterpart.

Finally, we have tried to link the anisotropy fields obtained with Kittel formula [9] with the magnetic parameters obtained after hysteresis loops. The figures calculated for anisotropy fields lie in the range of 0,8 to 1.7 KOe. This values are in the same order of magnitude that analog spinel ferrite or $\mathrm{Ni}$ ferrite nanoparticles synthetized with other methods [11-14], and roughly follow the evolution observed with the saturation magnetization so, unless there are other parameters which we do not have accounted for, like shape anisotropy that can also change in the series analyzed due to some degree of non-spherical shape or different degree of particle agglomeration, we can assume that crystalline anisotropy do not change abruptly with annealing temperature. Anyway, further research to elucidate some of the above mentioned hypotheses has to be carried out.

Finnancial support from Accion Integrada Hispano/Lusa AIB2010PT-00265 and Junta de Castilla y Leon Ref: VA230A11-2 is acknowledged.

\section{References}

1. H. Yang, X. Zhang, W. Ao, G. Qiu, Mater. Res. Bull., 39, 833 (2004)

2. J. Wang, Mater. Sci. Eng. B 127, 81 (2006)

3. ] R.D.K. Misra, S. Gubbala, A. Kale, W.F. Egelhoff Jr., Mater. Sci. Eng. B 111, 164 (2004)

4. A. R. Bueno, M. L. Gregory, M. C. S. Nóbrega, J. Magn. Magn. Mat. 320(6), 864 (2008)

5. U. R. Lima, M. C. Nasar, R. S. Nasar, M. C. Rezende, J. H. Araújo, J. F. Oliveira, Mater. Sci. Eng. B 151, 238 (2008)

6. T. Giannakopoulou, L. Kompotiatis, A. Kontogeorgakos, G. Kordas, J. Magn. Magn. Mat. 246, 360 (2002)

7. G.V. Kurlyandskaya, J. Cunanan, S.M. Bhagat, J.C. Aphesteguy, S.E. Jacobo, J. Phys. Chem. Solids, 68, 1527 (2007)

8. C.P.L. Rubinger, D.X. Gouveia, J.F. Nunes, C.C.M. Salgueiro, J.A.C. Paiva, M.P.F. Graça, P. André, L.C. Costa, Microwave and Optical Technology Letters. 49, 1341 (2007)

9. P. Hernández-Gómez, J.M. Muñoz, M.A. Valente, IEEE Trans. on Magn. 46(2), 475 (2010)

10. A.T. Raghavender, D. Pajic, K. Zadrob, T. Milekovic, P. Venkateshwar Rao, K.M. Jadhav, D. Ravinder J. Magn. Magn. Mat. 316, 1 (2007)

11. A. Kale, S. Gubbala, R.D.K. Misra, J. Magn. Magn. Mat. 277, 350 (2004)

12. H. Nathani, S. Gubbala, R.D.K. Misra, Mater. Sci. Eng. B 121, 126 (2005)

13. S. Thakur, S.C. Katyal, M. Singh. J. Magn. Magn. Mat., vol. 321, 1 (2009)

14. J. Azadmanjiri, S.A.S Ebrahimi, H.K. Salehani, Ceram. Intern., 33, 1623 (2007)

15. G.V. Kurlyandskaya, S.M.Bhagat, S.E.Jacobo, J.C.Aphesteguy, N.N.Schegoleva, J. Phys. Chem. Solids 72, 276-285 (2011)

16. R. Valenzuela, G. Alvarez, H. Montiel, M.P. Gutierrez, M.E. Mata-Zamora, F. Barron, A.Y. Sanchez, I. Betancourt, R. Zamorano, J. Magn. Magn. Mat. 320, 1961 (2008) 